\title{
SUPERCONDUCTING DRIVER LINAC BEAM DYNAMICS OPTIMIZATION FOR RIA*
}

\author{
D. Gorelov, J. Kim, F. Marti, H. Podlech, X. Wu, and R. York, \\ NSCL/MSU, East Lansing, MI 48824, USA
}

\section{Abstract}

The Rare Isotope Accelerator (RIA) facility is under study at NSCL [1]. A superconducting linac with total beam power of at least $100 \mathrm{~kW}$ and up to $400 \mathrm{~kW}$, capable of accelerating all light and heavy ions including uranium to the final energy of $400 \mathrm{MeV} / \mathrm{u}$ or more, is proposed as the driver accelerator for RIA. A multiple charge state beam acceleration concept [2] yields additional challenges for optimization of the heavy ion beam dynamics. Different variants of the linac layouts have been simulated using the LANA computer code [3]. Comparison of the simulation results for different options is presented.

\section{INTRODUCTION}

A major request from the experimental nuclear physics community is for a facility capable of producing radioactive nuclei beams with high energy and intensity [1]. The RIA project was proposed by the nuclear physics community as the next generation facility capable of producing beams of nuclei far from stability. This facility requires high power primary beams of stable light and heavy ions from hydrogen to uranium with energy 400 $\mathrm{MeV} / \mathrm{u}$ or more. A superconducting, high power, cw linac is considered as the driver accelerator for RIA.

A detailed study of the beam dynamics in the superconducting linac is underway [4,5].

\section{GENERAL LAYOUT OF THE DRIVER LINAC}

The general layout of the proposed driver linac for RIA (Fig. 1) includes ECRs, a room temperature RFQ, two stripping stations, and a superconducting linac.

The superconducting linac is divided by the stripping stations into 3 separate sections: low energy part from the RFQ at $\sim 170 \mathrm{keV} / \mathrm{u}$ to $\sim 13.5 \mathrm{MeV} / \mathrm{u}$, a medium energy part from $\sim 13.5 \mathrm{MeV} / \mathrm{u}$ to $\sim 85 \mathrm{MeV} / \mathrm{u}$, and a high energy part from $\sim 85 \mathrm{MeV} / \mathrm{u}$ to $\sim 400 \mathrm{MeV} / \mathrm{u}$.

\section{LONGITUDINAL BEAM DYNAMIC OPTIMIZATION}

The challenging feature of the proposed driver linac for RIA is the requirement to accelerate several charge states simultaneously.

Multi-charge acceleration increases the beam current accepted from the source and also mitigates the intensity loss at the strippers that are provided in order to reduce the total required voltage of the linac.

The longitudinal beam dynamics impose special requirements for configuration of the low energy part of the linac. The medium and high energy parts do not limit the longitudinal acceptance significantly.
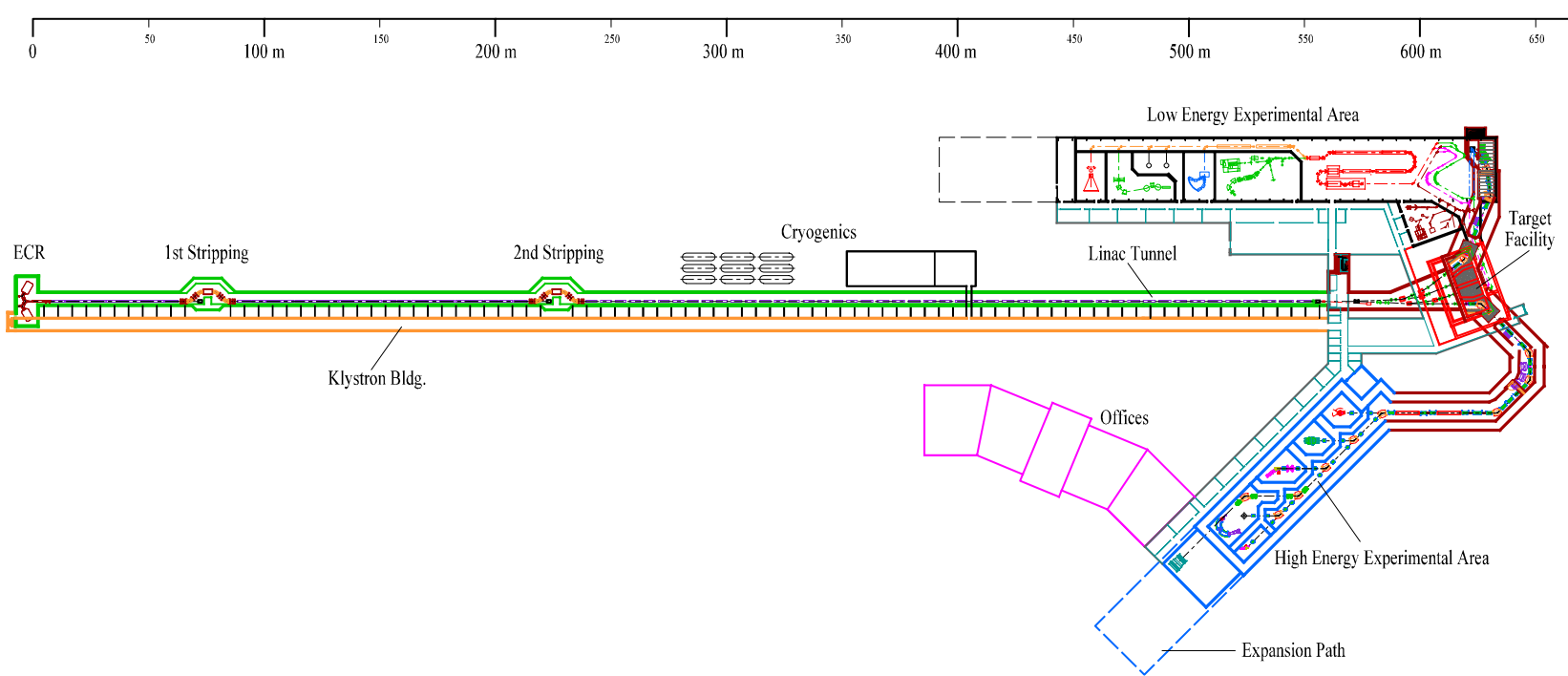

Figure 1: RIA facility layout. The driver linac extends from the ECR on the left to the Switch Yard in front of the Target Facility on the right. The linac consists of a room temperature RFQ and $\sim 450$ superconducting cavities in $\sim 100$ cryostats.

*Work supported by NSF Cooperative Agreement \# PHY-952844. 

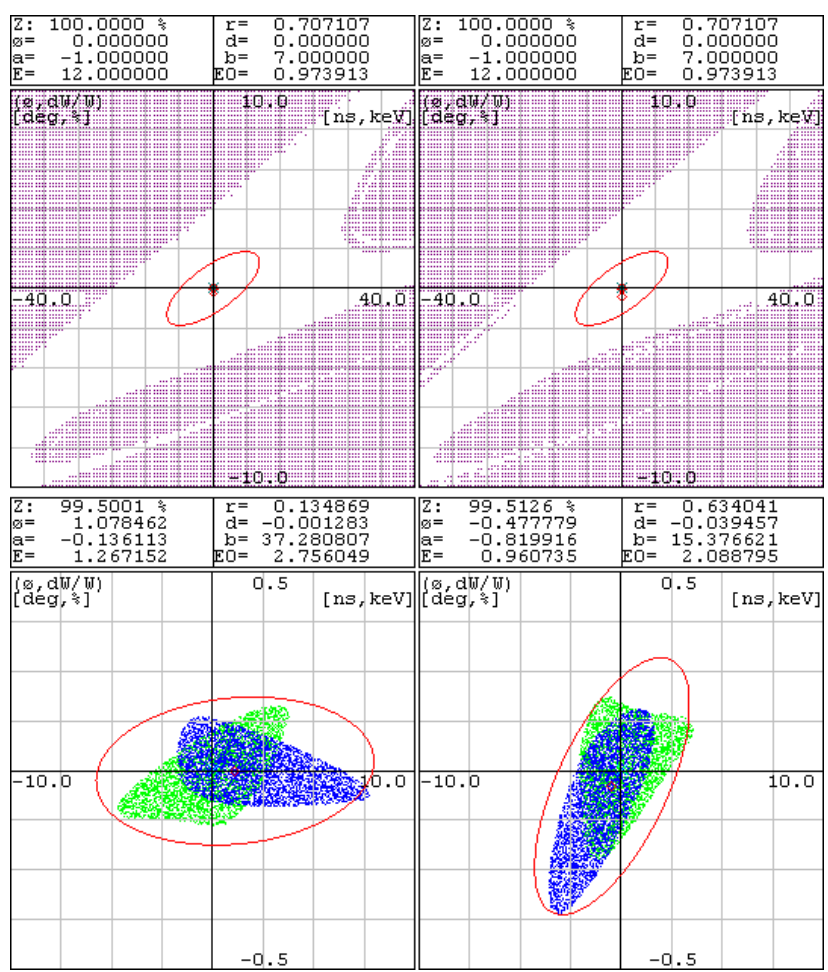

Figure 2: Longitudinal acceptance of the low energy part with initial longitudinal ellipses (top) and final distribution (bottom) of ${ }^{238} \mathrm{U}^{28,29+}$ for an equidistant layout (left) and for a special layout (right) of the cryostats.
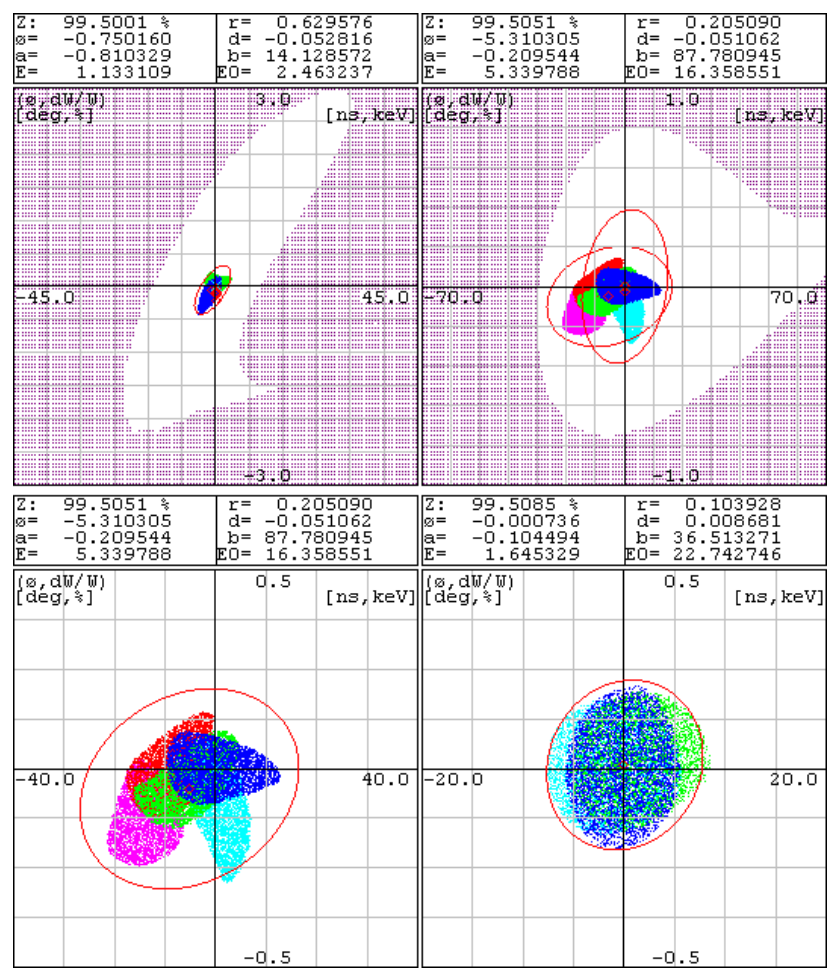

Figure 3: Longitudinal acceptances of the medium (left) and high (right) energy parts and final distributions of ${ }^{238} \mathrm{U}^{73-75+}$ and ${ }^{238} \mathrm{U}^{87-89+}$, respectively. The additional vertical ellipse in the upper-right-hand plot represents the matched initial distribution after the second stripper station used for the simulation.

\subsection{Low Energy Section Layout Optimization}

The low energy part of the linac extends from the output of the RFQ to the first stripper. The corresponding energy range is from $168 \mathrm{keV} / \mathrm{u}$ to $13.5 \mathrm{MeV} / \mathrm{u}$.

The longitudinal acceptance of this portion of the linac is shown in Fig. 2 in the top-left corner. Units are degrees at $57.5 \mathrm{MHz}$ and percent of the $168 \mathrm{keV} / \mathrm{u}$ input energy. The acceptance values correspond to the input emittance shown. The emittances in $\pi \cdot \mathrm{keV} / \mathrm{u} \cdot \mathrm{nsec}$ are also given for all cases.

The acceptance is calculated for a linac that consists of 100 low-beta superconducting cavities of 5 different types $(\beta=0.021-0.19)$ [4] grouped into 16 cryostats with a $50 \mathrm{~cm}$ drift space (from cold to cold) in between. Increasing the length of the drift space leads to a gradual deterioration of the acceptance. The final longitudinal distribution after acceleration in the linac (Fig. 2, bottom left) shows a significant increase (about 3 times) in the effective emittance of the combination of the two charge states, due to the difference in the longitudinal oscillation frequencies for the different charge states.

However, it is possible to find a layout that will provide less increase in the effective emittance ( 2 times) without a large decrease of the acceptance. The optimized layout was simulated, and the corresponding longitudinal acceptance and final distribution of the two charge states of a uranium beam are shown in Fig. 2 in the top right and bottom right corners, respectively. This layout has the same number of cavities and cryostats, but the distance between the cryostats is increased to $100-170 \mathrm{~cm}$ (depending on the phase of the longitudinal oscillations). Approximately $30 \mathrm{~cm}$ of this drift must be used to provide additional focusing. The remaining $70-140 \mathrm{~cm}$ (cold-tocold) could be used for convenient room temperature diagnostics and steering.

The disadvantage of this layout is a slightly increased number of focusing elements, and the necessity to fix the velocity profile of this portion of the linac for all the other ions by decreasing the accelerating gradient in proportion to the charge to mass ratio of the ion. This change does not affect the final energy for lighter ions in any of the cases that were simulated.

\subsection{Longitudinal Acceptances of Medium and High Energy Sections}

Fig. 3 shows the longitudinal beam dynamics simulation results for the medium and high energy parts of the linac.

The medium energy part between the stripping stations extends from 13.2 MeV/u (energy loss of $2 \%$ in the stripper is anticipated) to $88.8 \mathrm{MeV} / \mathrm{u}$. This section consists of 159 cavities of two types $(\beta=0.19-0.38)$ [4] grouped into 30 cryostats with $120-140 \mathrm{~cm}$ drifts in between.

Though the effective longitudinal emittance at the end of the medium energy section grows by about 5-6 times (Fig. 3, bottom left), no strategies were found to improve this situation because of the multiplicity of the charge 
states. Fortunately the acceptance of the high energy part (Fig. 3, top right) is sufficiently large to accommodate the resulting effective emittance. However, longitudinal matching at the exit of the second stripping station would be helpful.

The high energy part of the linac continues from the exit of the second stripping station to the end of the driver linac. The corresponding energy range is from 83.2 MeV/u (with $\sim 6 \%$ energy loss in the stripper) to $400 \mathrm{MeV} / \mathrm{u}$. This section consists of 3 types of elliptical cavities, one for $\beta=0.47$ and two SNS-like cavities for $\beta=0.61$ and $\beta=0.81$. The total number of cavities in this section is 184 and the number of cryostats is 59 . The general layout of the cryomodules and drift spaces was chosen to be similar to the SNS case [6]. The effective longitudinal emittance growth for the 3 simulated charge states is less then $40 \%$ in this section.

\section{TRANSVERSE FOCUSING SCHEME}

The results of $3 \mathrm{D}$ beam dynamics simulation with the realistic accelerating fields are presented in Fig. 4.

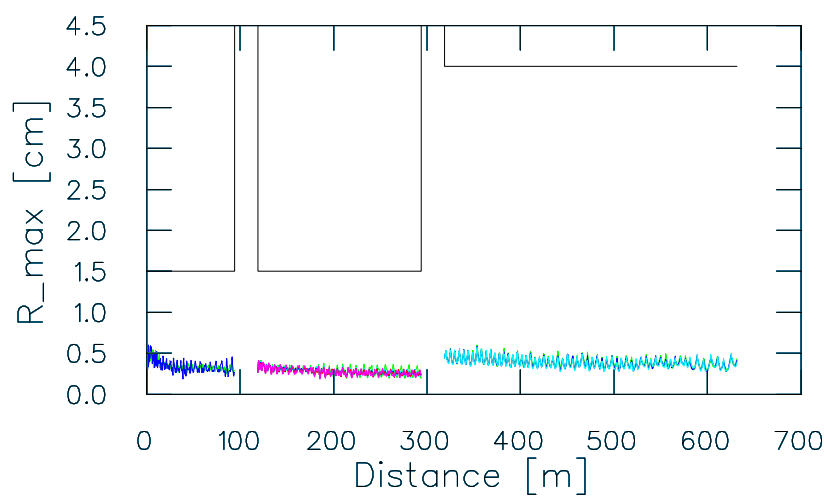

Figure 4: Maximal transverse envelopes for all simulated charge states of ${ }^{238} \mathrm{U}$ along the driver linac; the aperture of the respective superconducting cavities is also shown.

\subsection{Low and Medium Energy Section Focusing}

Transverse focusing with superconducting solenoids is used for the low and medium energy sections of the linac. The superconducting solenoids can be situated inside the cryomodules, and require a minimum of extra space. The limitations on the longitudinal dimensions of the linac make this type of focusing the most effective for the low and medium energy parts. The total number of superconducting solenoids used in the simulations was 136. The length varied from $10 \mathrm{~cm}$ in the first cryostat to $30 \mathrm{~cm}$ in the medium section. Most of the solenoids require a $\mathrm{B}$ field of 10 tesla or less and only 15 solenoids need to have a stronger field of up to 13 tesla. Technology exists for solenoids of 15 tesla and higher [7]. The transverse phase advance per focusing period $\sigma_{\mathrm{T}}$ is about $60^{\circ}$ for the simulations. This parameter was adiabatically ramped at the beginning and at the end of sections with different transverse focusing periodicity, in order to match the beam envelopes.

\subsection{High Energy Section Focusing}

Focusing with quadrupole doublets between cryostats (similar to those used for the SNS linac) was adopted for the high energy part. The total number of doublets needed for this section is 59 , with $R_{B O R E}=2 \mathrm{~cm}$, total length of a single quadrupole $\mathrm{L}=20 \mathrm{~cm}$, and field gradient $\mathrm{G}<56 \mathrm{~T} / \mathrm{m}$. The $\sigma_{\mathrm{T}}=60^{\circ}$ per focusing period with corresponding ramping between subsections with different periodicity proved to be optimal.

\section{PERFORMANCE OF THE LINAC FOR DIFFERENT IONS}

The driver linac was optimized for the most complicated case of ${ }^{238} \mathrm{U}$ ions. The linac performance for some other ions is summarized in Table 1.

Table 1: Charge states and energies $(\mathrm{MeV} / \mathrm{u})$ at the strippers and at the end of the linac for selected ions.

\begin{tabular}{|c|c|c|c|c|c|c|}
\hline Ion & $\begin{array}{c}\text { ECR } \\
\mathbf{Q}\end{array}$ & $\begin{array}{c}1 \\
\mathbf{Q}\end{array}$ & $\begin{array}{c}1 \\
\mathbf{E}\end{array}$ & $\begin{array}{c}\text { 2 str. } \\
\mathbf{Q}\end{array}$ & $\begin{array}{c}\text { 2 str. } \\
\mathbf{E}\end{array}$ & $\begin{array}{c}\text { Final } \\
\mathbf{E}\end{array}$ \\
\hline${ }^{1} \mathrm{H}$ & 1 & - & - & - & - & 929.6 \\
\hline${ }^{40} \mathrm{Ar}$ & 8 & 18 & 13.5 & - & - & 549.4 \\
\hline${ }^{136} \mathrm{Xe}$ & 18 & $47-49$ & 13.5 & 53,54 & 99.4 & 464.4 \\
\hline${ }^{238} \mathrm{U}$ & 28,29 & $73-77$ & 13.5 & $87-89$ & 88.8 & 400.5 \\
\hline
\end{tabular}

\section{CONCLUSION}

A new strategy for optimization of the longitudinal beam dynamics in the low beta superconducting linac has been proposed. A procedure for $3 \mathrm{D}$ matching of the different superconducting linac subsections has been developed and successfully applied to a specific driver linac layout.

Detailed studies of the stripping stations capable of matching the different charge states between sequential sections of the linac are required. Simulations of misalignment and $\mathrm{rf}$ jitter in the driver linac components are underway. These studies should determine the tolerances and layout of the steering elements.

\section{REFERENCES}

[1] http://srfsrv.jlab.org/ISOL/ ISOL Task Force Information.

[2] P.N. Ostroumov et al., "Multiple-charge Beam Dynamics in an Ion Linac", LINAC'2000, Monterey, August 2000.

[3] D.V. Gorelov and P.N. Ostroumov, "Application of LANA Code for Design of Ion Linac" EPAC'96, Sitges, June 1996.

[4] K.W. Shepard and T.E. Tretyakova, "Supeconducting Accelerating Structures for a Multi-Beam Driver Linac for RIA”, LINAC'2000, Monterey, August 2000.

[5] H. Podlech et al., these proceedings.

[6] R.L. Kustom, "An Overview of the Spallation Neutron Source Project”, LINAC'2000, Monterey, August 2000.

[7] http://www.cryomagnetics.com/. 